\title{
An Arithmetic Method for Mean Pulse Profile De-disperse of pulsars
}

\author{
X.J. $\mathrm{Wu}^{1,2}$ and X.F. $\mathrm{Liu}^{1,2}$ \\ 1 Beijing Astrophysics Center, CAS-PKU, Beijing 100871, China \\ ${ }^{2}$ Astronomy Department, Peking University, Beijing 100871, China
}

\begin{abstract}
In this paper we discuss an arithmetic method for mean pulse profile de-disperse. We proceed from the principle of dispersion measure, and invert the de-dispersion problem as to refutations of solving integral equations. Under idealization condition, the refutations results fit very well with the given theoretical curvature.
\end{abstract}

\section{Introduction}

Scattering, multipath propagation and the dispersion of pulsar radio pulse in the interstellar medium introduces distortion and time delay of pulse profile. The method to compensate the pulse profile for interstellar scattering was given by Kuzmin et al. (1993). The upper limit of band-width for single channel receiver is given by:

$$
B_{i}=\tau \times \nu_{M H z}^{3} / 8.3 \times 10^{3} \mathrm{DM}
$$

where $\tau$ is apparent beamwidth of mean pulse. For a certain filter-bank or correlation de-disperse system they can only fit for certain pulsars. The purpose of the method described in this paper is to compensate for the incomplete dedisperse on the pulsars. This method is also desired to enhance the ability of de-dispersion receivers.

\section{The de-disperse arithmetic method}

If we can divided the single channel receiver to multi-channel, then it is possible we can de-disperse them as in multi-channel receivers. On the assumption that $f\left(t, \nu_{s}\right)$ is the profile function obtained through a single channel receiver. The center frequency is $\nu_{s}$, band width is $\Delta \nu_{s} . f\left(t, \nu_{s}\right)$ is equivalent to many narrow band pulses superimposed without de-disperse. That is:

$$
f\left(t, \nu_{s}\right)=\sum_{i} \Psi_{i}\left(t-\Delta t_{i}, \nu_{i}\right)
$$

where $\Psi_{i}\left(t, \nu_{i}\right)$ is the pulses with sub-band width $\Delta \nu_{i}$ at center frequency $\nu_{i}$. Also we have $\sum_{i} \Delta \nu_{i}=\Delta \nu_{s} ; \Delta t_{i}=D M e^{2}\left(\nu_{i}^{-2}-\left(\nu_{s}+\Delta \nu_{s} / 2\right)^{-2}\right) / 2 \pi c m$, dispersions delay relative to the upper band of the receiver. The equation (2) transformed into:

$$
f(t)=\int_{\nu_{2}}^{\nu_{1}} \phi\left(t-A\left(\nu^{-2}-\nu_{1}^{-2}\right)\right) d \nu
$$


where $\nu_{1}=\nu_{s}+\Delta \nu_{s} / 2, \nu_{2}=\nu_{s}-\Delta \nu_{s} / 2, A=e^{2} D M / 2 \pi c m$.

The integral equation (3) can be segregated as:

$$
f(t)=\sum_{i=1}^{N} \Psi_{i}(t)
$$

So far the de-disperse refutations problem is simmer down to solve the integral Eq.(3) or Eq.(4). $\Psi_{i}(t)$ or $\phi(t)$ is the result to be refutated.

\section{Verify of the De-disperse Principle}

The pulse shape of mean profiles are shown as single peak, double peaks, triple peaks and multi-peaks. The single peak pulse has the feature of Gaussian distribution (Wu et al. 1993). Multi-peaks are also constructed by the independent Gaussian elements, which is widely adopted by previous works (Wu et.al.,1992,1998,Kramer,1994). We use the Gaussian elements and their composition to describe the different mean profiles (single peak, double peaks and three peak pulse). Presume a five channel receiver, the center frequency is $1000 \mathrm{MHz}$, total band width is $10 \mathrm{MHz}$ and $\mathrm{DM}=100 \mathrm{pccm}^{-3}$. Given the theoretical pulse curvature one of channel (width $2 \mathrm{MHz}$ ), make out the time delay of the five channels according the formula of the delay time for the dispersion, then we get the model pulse profile, add them together, we obtain the non-de-dispersed profile. Using the aforesaid method we refute the profile to see if we can deduce one channel's profile. This is aimed to verify if the arithmatic de-disperse has same effect as the de-disperse receiver. Under idealization condition, the refutations results fit very well with the given theoretical curvature.

The method of this paper can used to solve the previous observational data and also for the later observations.

Acknowledgments. This work is supported by NSF of China, the Climbing Program from the state Science and Technology Commission of China, and by RFDP Of China.

\section{References}

Kuzmin, A.D., \& Izvekova, V.A., MNRAS, 1993, 260, 724

Kramer, M.,1994, A\&AS, 107, 527

Wu, X.J. \& Manchester, R.N., Science of China, 1993, 136, 468

Wu, X.J., Xu, W. \& Rankin, J.M., Proc. IAU colloq., 128, ed. Hankin, Rankin \& Gil, 1992, 172

Wu, X.J., Gao X.Y., Rankin, J.M., Xu, W. \& Malofeev V.M., 1998 AJ. 116, 1984 